\title{
Oui, le Congo est un centre littéraire
}

Entretien mené par Claire Riffard et Sonia Le Moigne-Euzenot

Christian Gombo

\section{(2) OpenEdition}

12 Journals

Édition électronique

URL : https://journals.openedition.org/coma/5938

DOI : $10.4000 /$ coma.5938

ISSN : 2275-1742

Éditeur

Institut des textes \& manuscrits modernes (ITEM)

Référence électronique

Christian Gombo, «Oui, le Congo est un centre littéraire », Continents manuscrits [En ligne], 15| 2020, mis en ligne le 15 octobre 2020, consulté le 13 janvier 2023. URL : http://journals.openedition.org/ coma/5938; DOI : https://doi.org/10.4000/coma.5938

Ce document a été généré automatiquement le 13 janvier 2023.

\section{(c) (i) (9)}

Creative Commons - Attribution - Pas d'Utilisation Commerciale - Pas de Modification 4.0 International - CC BY-NC-ND 4.0

https://creativecommons.org/licenses/by-nc-nd/4.0/ 


\title{
Oui, le Congo est un centre littéraire
}

\author{
Entretien mené par Claire Riffard et Sonia Le Moigne-Euzenot \\ Christian Gombo
}

\section{NOTE DE L'ÉDITEUR}

Christian Gombo T. est écrivain et économiste de formation. Après plusieurs années dans différentes banques, il décide de tout abandonner lorsqu'il publie en 2017 Les Fables de Christian Gombo illustrées par Crebix Mozalisi aux éditions Nzoi, livre récompensé doublement au Mikanda awards 2017. Suit un roman en lingala, Bolingo eza n'a bozoba, aux éditions Mabiki (2018) et un recueil de poèmes, Maladies textuellement transmissibles, aux éditions Hans-kelsen (2019). Il est co-directeur artistique d'un projet annuel de création de «fanzines » initié par le Goethe-Institut, assistant de la présidente de l'ASBL Café Littéraire de Missy, assistant administratif et financier à ACRIA ASBL en partenariat avec le CALM ASBL pour l'organisation du « Salon Africain de la BandeDessinée et de l'Autre Muzik » (SABDAM 2019), traducteur de textes pour WWF DRC, lecteur et correcteur pour plusieurs éditeurs locaux et représentant officiel des éditions Nzoi en RDC. Membre du bureau de l'AJECO, Collectif Envie Écrire, ALEF, il travaille pour créer les conditions d'un marché rentable du livre localement en exploitant les spécificités du modèle économique congolais.

Claire Riffard et Sonia Le Moigne-Euzenot: Christian Gombo, vous êtes l'auteur d'un album, Fables, publié en 2017 aux éditions Nzoil à Kinshasa. Pouvez-vous nous donner quelques éléments sur la genèse de ce livre ? Sur la collaboration avec l'illustrateur, Crébix Mozalisi ?

Christian Gombo: Je suis économiste ruraliste de formation. Cette formation et, par la suite, mon travail de banquier m'ont donné la chance de visiter le Congo profond, l'arrière-pays. Témoin privilégié de plusieurs faits naturels, je les griffonnais dans mon carnet de voyage et petit à petit, ces histoires prenaient vie dans ma tête. D'autres histoires sont juste les réalités de ma vie quotidienne que je photographiais. Je ne parle pas beaucoup, j'aime observer, je suis très attentif au déroulement du quotidien. Cela me permet de visualiser le réel, c'est grâce à l'imagination que tout devient possible. 
Le rapport entre moi et Crebix Mozalisi vient du fait qu'il est un ami d'enfance devenu frère, avec qui on partage bien des rêves. Alors quand je lui ai parlé du projet, c'est comme s'il l'attendait déjà. Certaines de ses illustrations sont magiques et font mieux vivre le texte que les écrits. Un génie !

C. R. et S. L.M.-E. : Le genre littéraire de la fable nourrit l'imaginaire et propose des chemins de vertu. On en trouve dans tous les pays du monde. II a traversé les âges. Pourquoi écrire des fables en 2017 au Congo RDC et en français?

C. G. : La Fontaine a eu de l'influence sur moi. Je suis un grand lecteur de toutes ses fables et comme par hasard, on partage la même date de naissance ! J'ai lu Ésope aussi. Mon environnement culturel mêle fable et conte. Si j'aime tant la fable au point d'en publier un recueil, c'est parce qu'au début je voulais me faire comprendre d'abord des enfants car ce sont eux l'avenir. Le paysage littéraire de mon pays n'offre pas de grande visibilité à cette façon d'écrire alors même que c'est un genre littéraire pédagogique et si instructif. Je me suis lancé dans cette aventure pour me faire comprendre simplement par le plus grand nombre... Les fables permettent de dire beaucoup en peu de mots, elles suscitent le rire de quelques lecteurs. Je les ai écrites en français parce que je parle français de temps en temps et l'idée dans un premier temps était de les proposer dans les écoles.

C. R. et S. L.M.-E. : Justement, parlons un peu de la réception. Quel accueil a reçu votre livre?

Sur le plan commercial, cela a été un succès car tout le monde m'attendait avec ce livre. Ce n'est pas facile de vivre dans un environnement qui t'éloigne de ton rêve. J'écris depuis tout petit mais les possibilités de bien éditer n'existaient presque pas. C'est ce livre qui m'a lancé sur la scène littéraire congolaise. Le genre choisi, la fable, a joué, car c'était un genre "nouveau » qui a poussé bien des acheteurs à la curiosité. Tant mieux s'ils continuent de mordre à l'hameçon! Le livre a été primé deux fois aux Mikanda Awards 2017 et c'est toujours encourageant de voir l'enthousiasme que le livre suscite auprès de jeunes lecteurs...

C. R. et S. L.M.-E. : Vous parliez de votre désir de faire circuler votre livre dans les écoles. Pouvez-vous nous dire comment cela s'est passé? Votre démarche a-t-elle été de contacter les enseignants? Pouvez-vous nous raconter vos expériences dans le milieu scolaire? Ont-elles été possibles en milieu universitaire?

C. G. : À la parution de notre livre, nous avons eu le réflexe d'entamer des procédures pour que l'État s'approprie l'œuvre en la proposant aux écoles comme manuel utile pour l'Éducation nationale; en ce sens aussi j'ai personnellement rencontré certains hauts fonctionnaires du fonds de promotion culturelle, mais jusque-là, la lourdeur administrative a refroidi cet élan de motivation, on espère qu'il y aura des opportunités en ce sens bientôt...

Et même si l'État ne nous a pas encore répondu favorablement, nous ne sommes pas découragés pour autant. D'une certaine manière, on s'y attendait. Certains professeurs des écoles de la place ont acheté le livre après nous avoir vus lors de la phase de promotion du livre. Ils ont exploité certaines histoires dans un but pédagogique. Je cite le collège Boboto notamment où j'ai eu mon diplôme jésuite et mon diplôme d'État.

On a aussi pris l'habitude d'animer des activités culturelles en milieu scolaire, et de passer pour exposer et vendre à l'occasion de tous les évènements marquants qui 
réunissent des enfants; c'est dans de telles circonstances que nous croisons bien souvent des gens super motivés qui achètent le livre pour leurs propres enfants...

On est aussi allés exposer et vendre dans l'enceinte de l'Académie des Beaux-Arts, en nouant un partenariat avec un groupe de bédéistes qui ont créé un concept, «Zando $\mathrm{BD}^{2}$ ». L'idée est simple : chaque week-end, nous organisons un marché de vente de nos œuvres dans une école que nous avons ciblée à l'avance et ça marchait très bien jusqu'à l'arrivée de la Covid-19... Nous reprendrons certainement... et nous passerons dans davantage d'universités et d'écoles!

C. R. et S. L.M.-E. : On entend souvent dire que la littérature africaine occupe une position périphérique sur le marché mondial du livre, surtout lorsqu'il s'agit de littérature africaine francophone. Êtes-vous d'accord avec ce constat, vu de Kinshasa?

C. G. : Ne demandons pas à une tortue de courir plus vite que Usain Bolt! Il y a une réalité : le sous-équipement de l'Afrique, à cause surtout de certains de ses décideurs mais aussi des impérialistes mondiaux. Conséquence : peu d'États africains ont une conscience nationale, cela influe sur la production littéraire, et la misère constante n'aide en rien. Face au monde, notre littérature manque de voix car nous sommes cruellement privés de moyens pour la faire entendre, mais notre littérature existe et il revient à chacun de nous de lui donner les possibilités de sa progression sur le marché mondial. Je pense qu'on devrait se positionner à ce niveau.

L'erreur serait de croire que le marché mondial du livre peut se passer des Africains. L'Afrique possède une population jeune. Il est donc possible de créer d'abord un marché du livre local avant de nous placer en concurrence loyale avec les autres marchés. Nous devons réfléchir à développer notre propre marché avant toute chose.

L'existence de commerces liés au livre atteste de la rentabilité du secteur dans le temps. Il faut organiser ce secteur pour espérer un jour entrer sur le marché mondial. Quand bien même le moteur du livre tourne avec difficulté, les faits disent que les Congolais lisent, écrivent et achètent des livres, qu'ils vivent du livre... beaucoup de Congolais dépendent des revenus générés par le livre. Alors, faut-il nous pointer du doigt pour dire que notre littérature est dans une position périphérique? Sur le marché mondial du livre, des écritures congolaises remarquables écrasent bien souvent la concurrence, il y a des noms qui sont toute une littérature et je ne les citerai pas ici car ils sont mondialement connus et reconnus!

C. R. et S. L.M.-E.: "Les Congolais lisent, les Congolais écrivent». Acheter un livre représente un choix dans le budget quotidien. Pourriez-vous nous parler du prix du livre?

C. G. : En effet, les Congolais lisent. Parmi les écrivains résidant sur place, beaucoup sont d'excellents lecteurs, c'est déjà une base. À côté d'eux, il y a les populations. Il faut rappeler que les infrastructures de base nous font défaut pour avoir un accès facile à nos populations locales. Mais vous serez étonnés de découvrir que souvent les grands pools de vente se situent dans les périphéries de Kinshasa, au « Tshango » par exemple, qui a une grande population, et cette population s'intéresse au livre! C'est au "Tshango» qu'on peut sentir par exemple la popularité de Zamenga Batukezanga ${ }^{3}$, cet écrivain aujourd'hui décédé, très populaire à cause de sa façon simple d'écrire, qui traduisait au mieux les réalités locales.

Ces habitants dont on minimise souvent le potentiel de lecture sont attirés par tous les livres bon marché qui parlent de leur réalité quotidienne. Des fanzines, des bd locales, des livres imprimés à moindre coût circulent et se vendent comme des petits 
pains. À côté de ces ventes d'ouvrages littéraires de petits formats, il y a des bouquinistes avisés qui vendent selon les capacités financières locales, et pour finir des libraires plus traditionnels qui y sont aussi installés, comme la librairie des éditions Mabiki.

La présence de tous ces vendeurs de livres atteste de la rentabilité du secteur même quand le livre est de format modeste. Généralement, en ce genre de lieu, la moyenne de prix tourne autour d'un euro. Mais il y a aussi quantité de livres venus d'ailleurs, que les grands libraires importent et vendent bien souvent dans et autour du centreville. Généralement la moyenne du prix d'un livre importé tourne autour de $10 €$, et cela ne réduit pas la capacité de vente des libraires. Dans ce secteur, on trouve les éditions Elondja ${ }^{5}$, Médias Paul ${ }^{6}$, Paulines ${ }^{7}$, Harmattan ${ }^{8}$, Librairie Book express, Librairie Grands Lacs ${ }^{9}$... Ce sont de vraies entreprises, qui payent leur personnel grâce aux ventes de livres principalement.

C. R. et S. L.M.-E. : Le marché local du livre congolais serait-il donc en expansion rapide?

C. G. : Je peux dire en étant sûr de n'être pas contredit que la taille du marché est grande, et que les acteurs du livre n'arrivent pas encore à satisfaire entièrement la demande. Comme déjà dit, ce sont des communes « reculées » de Kinshasa qui offrent les meilleures possibilités de vente quand le prix est abordable et que les ouvrages parlent de la réalité des habitants. Quant aux libraires traditionnels, les bouquinistes et les vendeurs ambulants assurent constamment la vente de tous types d'ouvrages dans les grandes villes et au centre-ville de Kinshasa. La population de la RDC est estimée à plus de 70 millions d'habitants dont près de 10 millions vivent à Kinshasa. Un potentiel énorme, et presque inexploité. Et il faut ajouter que plusieurs associations culturelles écoulent les livres de leurs membres en organisant différents types d'activités presque partout à Kinshasa.

Néanmoins, il reste encore du travail à faire pour combler entièrement la demande de livres et créer un modèle de vente local qui fera du pays un énorme marché, vu la taille de sa population...

C. R. et S. L.M.-E. : Quel serait ce modèle de vente local ?

C. G. : J'ai parlé des spécificités du marché local congolais. Au pays, les acteurs du livre ne sont pas soutenus par l'État. Produire pour vendre ou revendre reste un acte courageux. Le pays est vaste et manque cruellement d'infrastructures adéquates pour la distribution et la diffusion des livres, on ne peut donc pas vendre comme partout ailleurs dans le monde. Les écrivains sont alors contraints à être les premiers agents marketing de leur livre, et à côté de cela, le livre doit être à proximité de tous les acheteurs potentiels. C'est ce que beaucoup d'associations essayent de faire en multipliant des évènements culturels un peu partout dans le pays.

Ainsi donc, on trouve des livres partout : vente aux étalages de supermarchés, chez les bouquinistes où ils sont étalés par terre presque partout dans le pays, vente dans quelques restaurants bien cotés (un modèle qui a bien fait vendre mes fables), vente directe dans les écoles (Zando BD), vente ambulante organisée, vente dans les salles de fêtes (mariages, anniversaires dans les sphères riches), vente en ligne avec possibilité de livraison à domicile en exploitant les réseaux sociaux, vente de livres en format pdf via Internet avec des paiements en mobile Money, vente dans les foires agricoles, industrielles, minières... Bref, tout rassemblement peut être l'occasion de 
croiser des lecteurs potentiels et, une fois que ce modèle de vente sera bien organisé, ce sera une base solide sur laquelle reposera la rentabilité du livre.

C. R. et S. L.M.-E. : À ce propos, quel nouveau système de diffusion envisageriez-vous de mettre en place pour/avec Nzoi, votre éditeur?

C. G. : Pour la distribution et la diffusion des livres Nzoi, j'exploite tous les rendezvous culturels majeurs du pays sans oublier de présenter régulièrement les ouvrages dans les espaces de rencontres littéraires, au Café littéraire de Missy ${ }^{10}$ (le CalM) par exemple, qui me permettent d'exposer et de vendre.

Fig. 1 : Christian Gombo dans une salle de classe modérant une rencontre avec l'écrivaine suisse Anne-Sophie Subilia

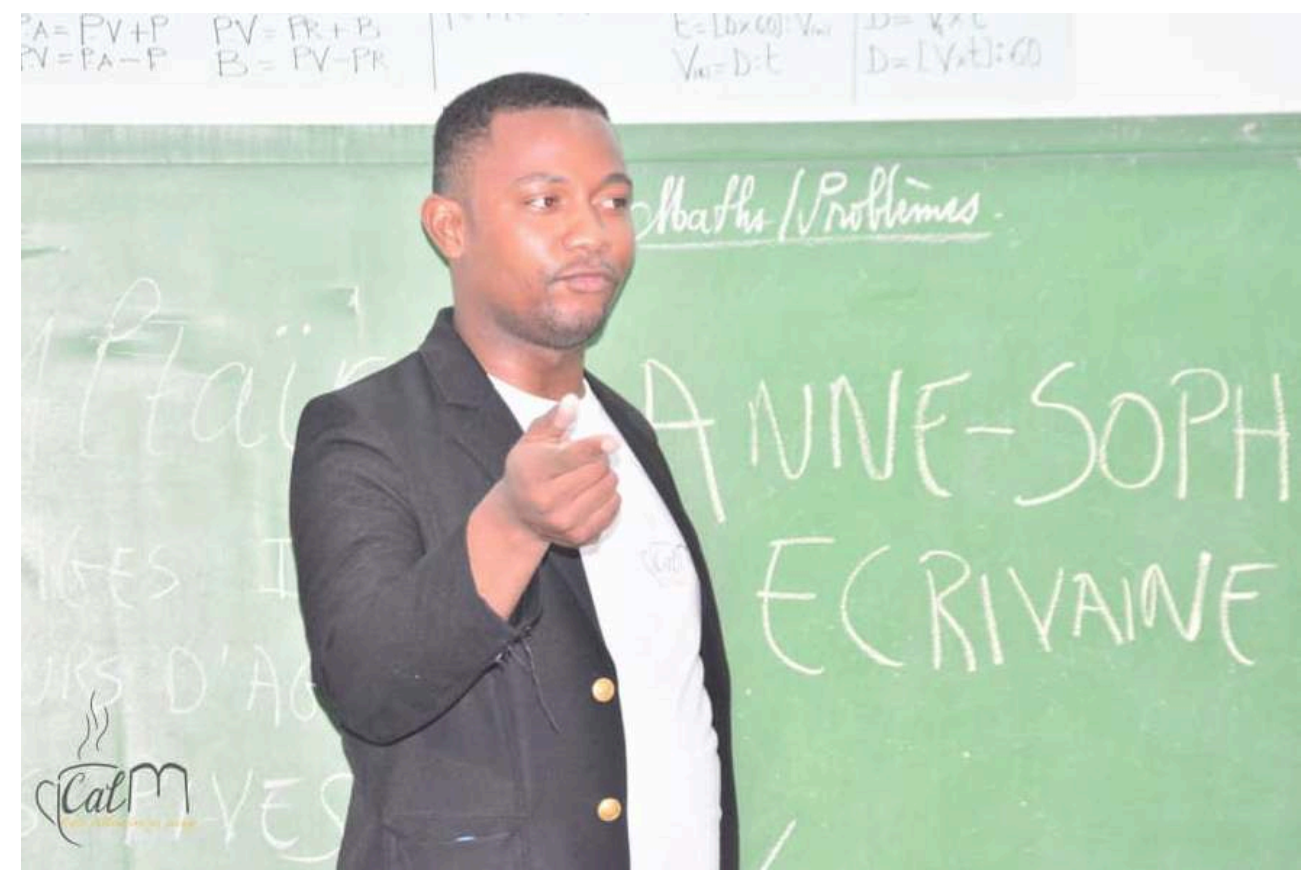

Café littéraire de Missy

Je suis aussi en train de créer un modèle économique du marché du livre congolais qui tient compte de la spécificité du marché local du livre. Ce modèle économique va intégrer différents réseaux: vendeurs ambulants, vente directe dans les écoles et universités, bouquinistes, libraires traditionnels, quelques restaurants et supermarchés, quelques lieux clés comme l'aéroport, etc. 
Fig. 2 : Stand de livres à l'occasion d'un salon

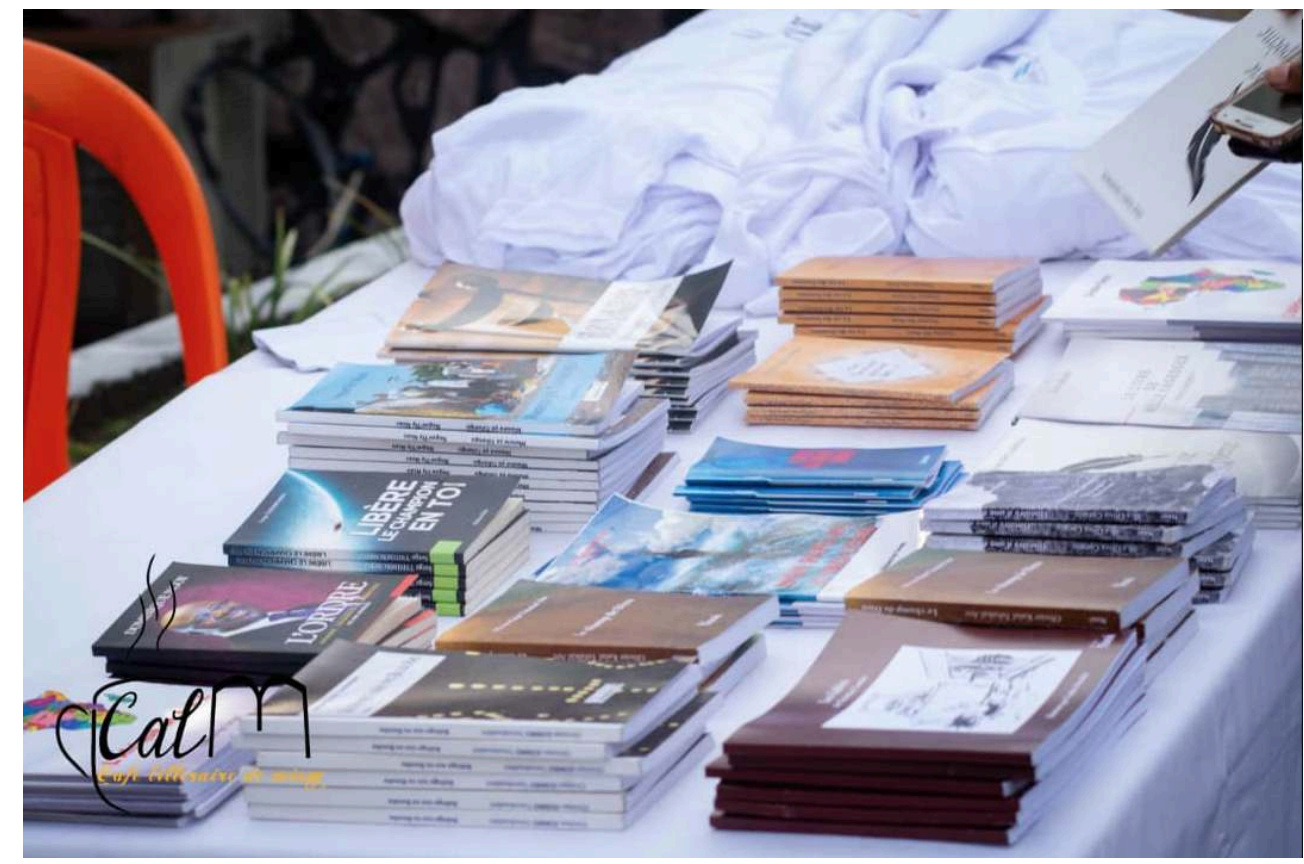

Café littéraire de Missy

C. R. et S. L.M.-E.: Vous avez évoqué les associations littéraires et culturelles, les restaurants... Quelles structures permettent aujourd'hui une vie du livre en RDC ? Quelles sont les dynamiques à l'œuvre, selon vous?

C. G. : Il y a plusieurs structures qui font vivre les livres en RDC; le pays est grand, je ne saurais pas toutes les citer.

Il $\mathrm{y}$ a déjà le CalM où je suis l'assistant de la coordinatrice Missy Bangala; il y a le Tarmac des auteurs ${ }^{11}$, il y a le groupe Envie d'écrire présidé par le poète Lyricomane $^{12}$. Il y a l'ALEF (Atelier pour le leadership et l'excellence dans la formation ${ }^{13}$ ) dont Alfred Dibandi fait partie de l'équipe dirigeante; il y a l'AJECO (l'Association des jeunes écrivains congolais ${ }^{14}$ ) présidée par Djodji Belau; il y a le centre Wallonie-Bruxelles International ${ }^{15}$; le Goethe-Institut ${ }^{16}$; l'Institut français de Kinshasa ${ }^{17}$, etc. L'ensemble du travail de tous ces acteurs rend compte de l'actualité littéraire, sans ignorer d'autres moyens liés aux réseaux sociaux qui rapprochent lecteurs et auteurs. 
Fig. 3 : Christian Gombo interviewé par Nioni Masela pour le compte de Dépêches de Brazzaville

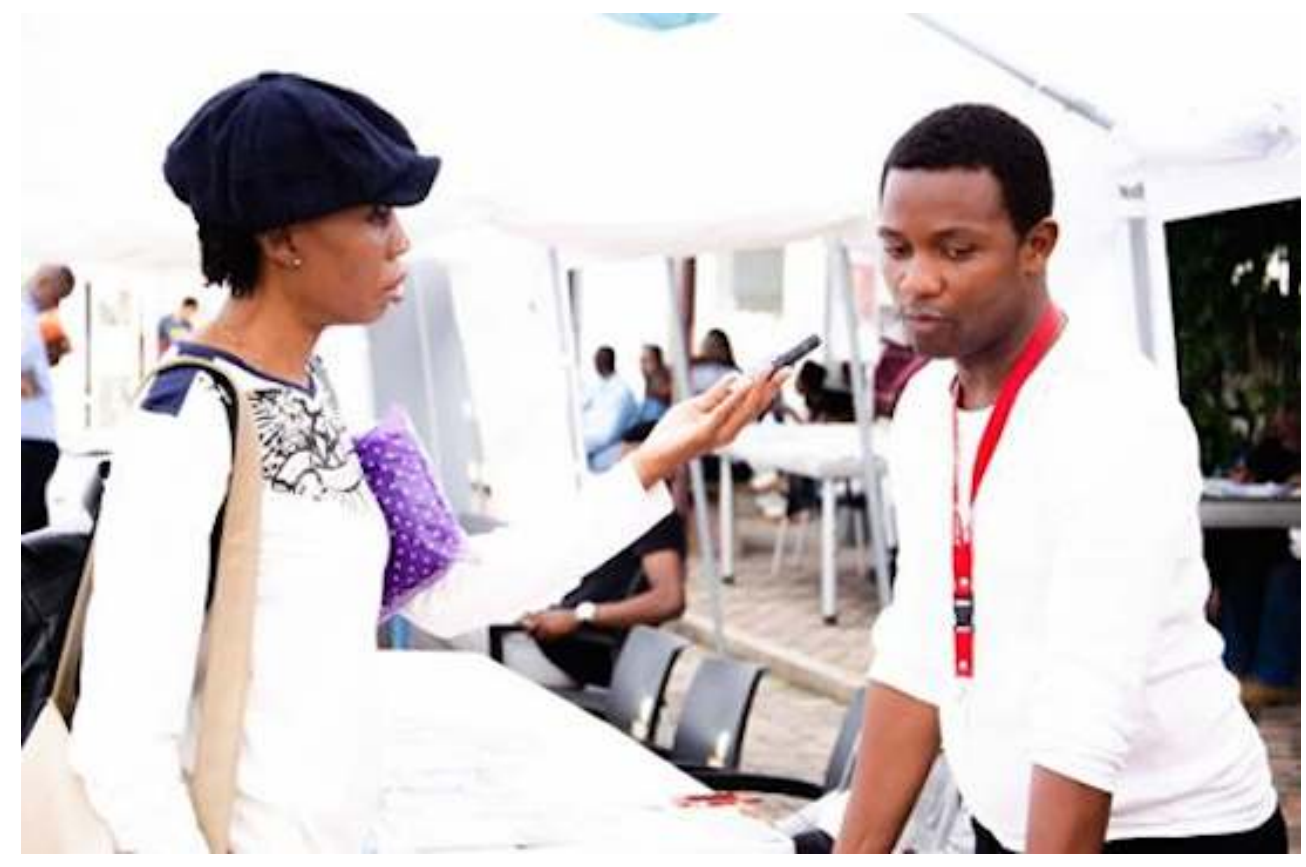

Dépêches de Brazzaville

Fig. 4 : Participation de Christian Gombo à un salon littéraire

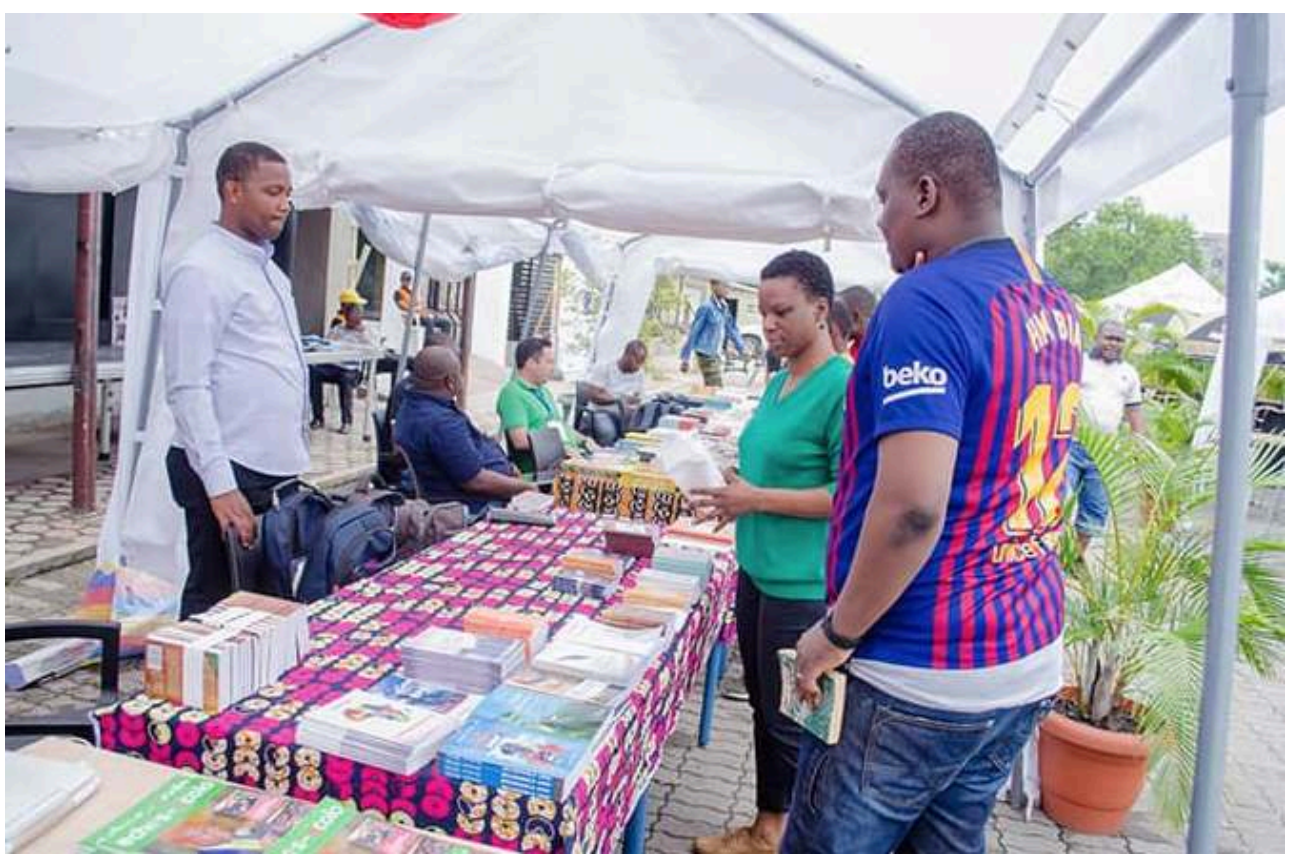

???

Les journalistes littéraires qui travaillent pour les médias du pays sont des gens à féliciter et à encourager. Avec le temps, les médias s'interrogent de plus en plus sur la littérature et des tas de passionnés essayent de donner aux auteurs des espaces de liberté pour la promotion de leur ouvrage. Toutefois, leur travail se fait souvent dans des conditions difficiles et petit à petit des voix s'élèvent pour honorer ces journalistes qui contribuent à leur façon à l'essor de la culture congolaise. 
Évidemment, leur rôle est incontournable pour la promotion du livre et des auteurs, et de plus en plus ils sont invités à couvrir la plupart des événements culturels que nous nous efforçons de maintenir. Que ce soit à la radio ou à la télé et mêmes sur Internet, il y a de plus en plus d'émissions qui rendent compte de l'actualité littéraire. Actuellement, plusieurs blogs sont presque entièrement dédiés à la littérature. Je peux citer celui de Fady Ambroise ${ }^{18}$, "Le monde de $\mathrm{Fa}^{19}$ ", le site de Soraya Majuscaux ${ }^{20}$, «Au car de Tour ${ }^{21}$ » de Tata N'longi Biatitudes où, en quelques minutes, dans une voiture, il interroge un auteur; quant aux sites internet, on peut citer celui du Café littéraire de Missy, et tant d'autres voués à la culture congolaise en général ${ }^{22}$.

C. R. et S. L.M.-E. : Vous avez cité Tata N'longi Biatitudes, qui a aussi publié aux éditions Nzoi un ouvrage récent, Bateki Mboka. Y aurait-il actuellement l'émergence d'une nouvelle génération d'écrivains à Kinshasa? Ont-ils des liens entre eux? Se sentent-ils reliés dans leur manière de faire et de vivre la littérature?

C. G. : Avant il était difficile de partager sa passion du livre avec d'autres passionnés du livre. Les réseaux sociaux et les structures dont j'ai déjà parlé ont fortement contribué à cette évolution. Les écrivains et d'autres acteurs du livre ont plusieurs rendez-vous incontournables liés à ces structures. Une nouvelle génération d'écrivains à Kinshasa prend plaisir à exister et à faire entendre sa voix. Des liens s'affermissent, se consolident pour une littérature plus vivante que jamais.

C. R. et S. L.M.-E. : Pensez-vous que le Congo puisse être considéré comme un centre littéraire?

C. G. : Oui, je le pense ! Rares sont les pays où la rentrée littéraire est organisée en plein centre-ville, où la fête du livre a un écho mondial! Ajoutez à cela différents festivals haut en couleur destinés à faire vivre le livre (FELIBI ${ }^{23}, \mathrm{SABDAM}^{24}$, etc.). Oui, le Congo est un centre littéraire. Des passionnés de lecture, d'écriture et de culture n'abandonnent pas, alors que tout est compliqué. Cette résistance en soi en dit long sur le travail abattu par chaque intervenant du livre. Encore une fois, oui, le Congo est un centre littéraire. Kinshasa est depuis un moment une grande ville du livre et le travail fourni en ce sens mérite le respect de tous ! 
Fig. 5 : Signature de Christian Gombo à un salon du livre

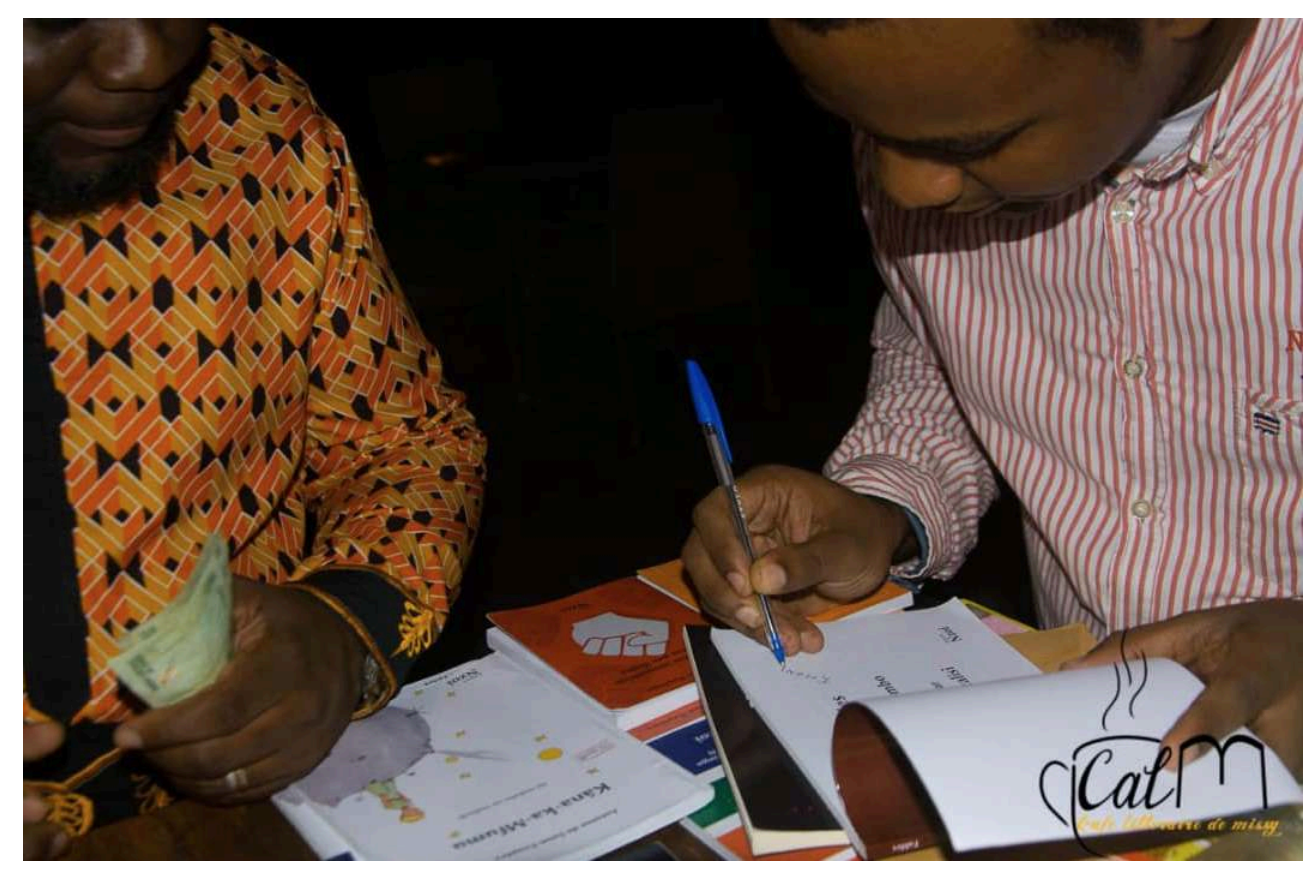

Café littéraire de Missy

\section{NOTES}

1. Créées en 2012, les éditions Nzoi entendent contribuer à la diffusion des livres à Kinshasa d'abord, puis dans le reste de la République démocratique du Congo (RDC). Elles s'intéressent à tous les genres littéraires : roman, récit, nouvelle, poésie, formes slamées, théâtre, essai, travaux de sciences sociales, bande dessinée. L'idée est de faire connaître sur place et à l'étranger ce qui s'écrit en RDC, de publier des textes de qualité, rédigés par des auteurs et des autrices de RDC et, plus largement, des Suds, écrits en français mais aussi dans les quatre langues nationales de la RDC, le lingala (deux ouvrages publiés), le kikongo (un ouvrage publié et un deuxième en préparation pour 2020), le ciluba (un ouvrage) et le swahili (aucun ouvrage à l'heure actuelle), imprimés en RDC, à des prix abordables (dans la grande majorité, entre 4000 et 25000 francs congolais, soit approximativement entre deux et quatorze euros) et destinés à un large public. Publient environ trois à quatre livres par an. Voir http://www.editions-nzoi.org.

2. En ligne sur facebook.com.

3. En ligne sur africultures.com.

4. En ligne sur makibi.net.

5. Maison d'édition fondée par Dan Bomboko. Voir afribd.africultures.com.

6. Voir éditions mediaspaul.fr.

7. Voir editions.paulines.qc.ca.

8. Voir editions-harmattan.fr.

9. Voir livresgrandslacs.com.

10. Voir cafelitterairedemissy.com. 
11. Voir tarmacdesauteursblog.com.

12. Voir facebook.com.

13. Voir twitter.com.

14. Voir ajecordc.blogspot.com.

15. Voir wbi.be.

16. Voir goethe.de.

17. Voir institutfrancais-kinshasa.org.

18. Voir fadyambroise.com.

19. Voir lemondedefa.com.

20. Voir majuscaux.wordpress.com et youtube.com

21. Voir facebook.com.

22. Voir culturecongo.com et arts.cd.

23. En ligne sur culturecongo.com.

24. Voir youtube.com.

INDEX

Mots-clés : fable, circulation des livres en milieu scolaire, place de la littérature africaine, reconnaissance de la littérature congolaise, marché du livre au Congo

\section{AUTEUR}

CHRISTIAN GOMBO

Écrivain et économiste rural congolais, né à Kinshasa en 1988 